86 ALÉTHEIA

2014, 2(1) 86-99

Recibido: 15-04-2014

Aceptado: 20-08-2014

Palabras clave: componentes linguisticos, comprensión lectora.

Keywords: linguistic components, reading comprehension.

Hilda Díaz Sánchez I.S.P. María Auxiliadora alhetia7@hotmail.com Angie Elisene Pintado Negron Colegio Reina del Mundo angiepintado_ps@yahoo.es Sandra Alicia Salas Condori

Consultora en Educación ciandra24@hotmail.com

\section{Relación entre los componentes lingüísticos y el nivel de comprensión lectora en estudiantes del sexto grado de educación primaria}

\section{Relationship between linguistic components and reading comprehension's level in sixth grade primary students}

\section{Hilda Díaz Sánchez \\ Angie Elisene Pintado Negron \\ Sandra Alicia Salas Condori}

RESUMEN. La presente investigación es de nivel descriptivo no experimental de tipo sustantiva, con diseño correlacional. El objetivo principal fue determinar la relación entre el nivel de los componentes lingüísticos y el nivel de comprensión lectora. La muestra de estudio estuvo conformada por 271 estudiantes de tres instituciones educativas de tipo estatal, parroquial y particular. Se utilizó la prueba CLP de Compresión Lectora de Condemarin y Milicic adaptada por Delgado y Cols. Para evaluar el nivel de los componentes lingüísticos se usó la Batería de Lenguaje Objetiva y Criterial BLOC; adoptada por Panca y Cols. Se concluyó en que existe correlación entre el nivel global de los componentes lingüísticos y el de comprensión lectora, según tipo de institución educativa y género en estudiantes del sexto grado de primaria.

ABSTRACT. This research is of non-experimental descriptive level of substantive type, with correlational design. The main objective was to determine the relationship between the level of linguistic components and the level of reading comprehension. The study sample consisted of 271 students of three educational institutions of public, parochial and private type. Progressive Linguistic Complexity Test of reading comprehension of Condemarin and Milicic was used, adapted by Delgado and collaborateurs. To assess thelevel of linguistic components, the Objective Criteria Language Battery (BLOC) was used; adopted by Panca and collaborateurs. It was concluded that there is correlation between the overall level of linguistic components and the level of reading comprehension, according to type of eductional institution and gender in sixth grade students of primary education.

\footnotetext{
* Tesis para optar el Grado de Maestría en Educación, UNIFE, 2014
} 


\section{Introducción}

$\mathbf{E}$

1 lenguaje como instrumento permite el desarrollo de las personas, su estudio resulta ser complejo, por estar constituido de cuatro componentes que son: morfológico, sintáctico, semántico y pragmático que de no ser bien construidos o formados desde la infancia puede acarrear dificultades en otros aspectos como la lectura y específicamente en el proceso de la comprensión lectora.

..., el conocimiento y el lenguaje son herramientas básicas en el razonamiento, tanto inductivo como deductivo. Muchos de los errores mostrados por los niños pequeños en las tareas de razonamiento no se deben a un pensamiento ilógico, sino más bien a errores en la interpretación de los problemas a resolver, es decir, a un desconocimiento del lenguaje en algunos de sus componentes (sintáctico, semántico, morfológico...)(Muñoz,2010,p.109).

Por otro lado, llama la atención los resultados de evaluaciones nacionales (UMC 2009) e internacionales (PISA 2000) que evidencian el bajo nivel de comprensión lectora de los estudiantes de nuestro país. Los resultados de la Unidad de Medición de la Calidad 2009, expresa que el 58,2 \% no comprende lo que lee y el $28,2 \%$ comprende textos breves o partes de un texto en los niños de cuarto grado de primaria.

De este modo los objetivos de esta investigación son dos: primero, determinar la relación entre el nivel global de los componentes lingüísticos y el nivel de comprensión lectora en estudiantes del sexto grado de educación primaria, de diferentes Instituciones Educativas (estatal, parroquial y particular) y segundo, determinar la relación entre el nivel global de los componentes lingüisticos y el nivel de la comprensión lectora en estudiantes del sexto grado de educación primaria, según género.

Con respecto a anteriores investigaciones, se encuentran la de Blondet y Pinheiro (2012) (Universidad Femenina del Sagrado Corazón- Unifé), quienes realizaron un estudio denominado Efectos del programa CLO para mejorar las componentes del lenguaje oral expresivo en niños con trastornos específicos del lenguaje (TEL), diseñado desde un enfoque pedagógico. La muestra estuvo conformada por 8 niños con TEL de 10 a 12 años que asisten al 5to y Gto grado de primaria. Se partió de la hipótesis que el programa "CLO", mejora los componentes del lenguaje oral expresivo, morfológico, sintáctico, semántico y pragmático en niños con TEL de 5to y 6to grado de primaria. Se aplicó a ambos grupos el pre-post test con la prueba "Batería del lenguaje objetiva y criterial BLOC-S", además antes del inicio del programa se recopilaron los informes psicopedagógicos de cada estudiante para determinar por criterio de exclusión que presentaban el trastorno especifico del lenguaje.

$\mathrm{Al}$ final del estudio se pudo confirmar que se reportaron mejoras en todos los componentes del lenguaje oral. Esta evidencia sugiere una consideración del programa "CLO" como un potencial método de enseñanza para niños de 5 to y $6^{\circ}$ grado de primaria diagnosticado con TEL.

Realizando una indagación sobre lo que podrá influir en la comprensión lectora aparece el lenguaje y sus componentes. El lenguaje, es un instrumento básico y necesario que le permite relacionarse con sus semejantes, es un acto de comunicación que posibilita expresar y comprender ideas, pensamientos y sentimientos, constituye una herramienta indispensable en la vida del hombre y su estudio es de suma importancia. Por esta razón, es necesario comprender cómo se adquiere y desarrolla, qué teorías importantes son las que explican su adquisición y finalmente cuáles son sus componentes.

En cuanto a su adquisición, Miretti (2006) afirma que se realiza en dos grandes etapas: etapa prelingüística (enmarcado en el primer año de vida) y la etapa lingǘstica (aproximadamente entre el año, año y medio).

La etapa prelingüística corresponde al momento evolutivo de aprestamiento, previo al inicio del lenguaje. Se desarrolla en el primer año de vida y comprende las primeras vocalizaciones (gritos, llantos y ecolalia), el balbuceo y la expresividad gestual. 


\section{UNIFÉ - EPG}

Esta etapa es de suma importancia por lo que Miretti afirma "que tiene un valor relevante y trascendental en la configuración de las bases del desarrollo lingüístico, puesto que esas expresiones vocales son determinantes en el desarrollo posterior de la comunicación lingüística del niño" (2006, p. 26).

De igual importancia, la etapa lingüística se inicia aproximadamente durante el primer año de vida con la expresión de la primera palabra del niño y se caracteriza por los siguientes hechos observables: Dominio fonológico, aparición de primeras palabras, incremento del vocabulario, aparición de holofrases que luego constituirán enunciados y la adquisición y aplicación de reglas gramaticales.

$\mathrm{El}$ incremento del vocabulario va deberse específicamente al factor de sociabilización que tiene el niño por coger el mundo, su curiosidad y deseo de aprehender todo lo que le rodea. Niño (2002), afirma que "La época de las preguntas no es otra que una manifestación del deseo de coger el mundo y socializarse, en tanto que se tranquiliza con las respuestas del adulto.” (p. 19).

Miretti (2006), por su lado indica que “... la identificación y denominación de objetos, figuras y diferentes partes del propio cuerpo, son ejercicios muy recomendables para el desarrollo del lenguaje verbal del niño" (p. 31).

Así también el inicio a la etapa escolar va constituir una fase de afianzamiento, ampliación y concientización de las estructuras lingüísticas adquiridas.

Después de los cinco o seis años, el niño avanza hacia la socialización y entra en una fase de afianzamiento, ampliación y concientización de las estructuras lingüísticas ya adquiridas, a través del desarrollo de las habilidades comunicativas (entre las que cuenta la lecto-escritura), acontecimientos que coinciden con la vida escolar (Niño, 2002, p. 21).

Sin embargo, en la tarea que el niño tiene de incrementar su repertorio de palabras, va cometiendo errores que el adulto debe ir corrigiendo con bastante tino, discreción y sin censura puesto que los comete sin darse cuenta como parte de su proceso de desarrollo. "La labor correctiva, o mejor, la aprobación o desaprobación de los mayores es más efectiva y menos traumatizante si se apoya con el ejemplo y no en la censura en sí misma” (Niño, 2002, p .19).

Por otra parte, a través de los tiempos han surgido dos grandes corrientes filosóficas que intentan explicar cómo nació el lenguaje: la innatista que sostiene que el lenguaje es un don biológico con el cual nacen los humanos, y la empirista que defiende que el entorno social es el único factor determinante en el desarrollo idiomático.

De ambas corrientes se desprenden las aportaciones de las principales teorías de adquisición del lenguaje, teorías que no necesariamente se contraponen sino que en algún momento del desarrollo humano, interactúan y se complementan. Así mismo, Berk (1999) sostiene que "Como consecuencia, hoy hay un reconocimiento de que la biología, cognición y la experiencia social pueden funcionar de diferentes formas con respecto a cada componente del lenguaje" (p. 470).

Conocer y emplear correctamente el lenguaje implica, conocer e interactuar sus componentes que son: fonología, morfología, sintaxis y semántica, así como su pragmática.

Cuando el ser humano emplea el lenguaje, hace uso de cada uno de estos componentes quienes a su vez se ven regidos por las reglas gramaticales quienes indican y dirigen la forma correcta de utilizar las palabras, mientras que el agravio a estas reglas produciría frases agramaticales.

Con ello se puede decir que el hablante que conoce y utiliza adecuadamente cada uno de los componentes del lenguaje ha adquirido competencia comunicativa tal como lo señala Hymes (1972) cuando afirma que:

tenemos que explicar el hecho de que un niño normal adquiere conocimiento de las oraciones, no sólo como gramaticales, sino como apropiadas. Él o ella adquiere competencia en relación a cuándo hablar, cuándo no, así como de qué hablar con 
quién, cuándo, dónde y de qué manera

(citado por Galeote, 2002, p. 20).

De este modo, el proceso de adquisición del lenguaje implica lograr no sólo competencia lingüística, sino también competencia comunicativa

Desmenuzando cada uno de estos componentes por índole de estudio, Acosta y Moreno (1999) sostienen que la fonología se interesa por el estudio de la organización de los sonidos en un sistema valiéndose de sus caracteres articulatorios y de la distribución o suma de los contextos en que pueden aparecer. Lo que indica que la fonología intenta entender la influencia que los sonidos tienen sobre otros, dando lugar a los datos fonéticos y analizando elementos que permitan reconocer el mismo sonido.

Owens (2003, p. 18) señala que "La morfología tiene que ver con la organización interna de las palabras y la unidad mínima de significados que existe en una lengua se denomina morfema: la unidad gramatical más pequeña".

BerkoyBernstein (1999), definenelcomponente sintáctico como "el estudio de la combinación de palabras para construir oraciones de forma correcta en una lengua" (p. 18).

Tal es así que la gramática es la parte que describe la estructura del lenguaje e incluye reglas para combinar palabras en la formación de frases u oraciones.

Berko (1993), (citado por Acosta, 1999 p. 107) refiere que "la semántica abarca los procesos de codificación y decodificación de los significados del lenguaje". En tal sentido, respectivamente hace referencia a la comprensión del lenguaje; es decir, extraer el significado a partir de nuestro sistema simbólico; expresamente, supone una selección apropiada del vocabulario y estructura del lenguaje para transferir significado, lo cual depende del contenido y propósito de lo que se quiera comunicar.

$\mathrm{Y}$ finalmente la pragmática, estudia el funcionamiento del lenguaje en contextos sociales, situacionales y comunicativos, es decir analizan las reglas que regulan el uso intencional del lenguaje, teniendo en cuenta que se trata de un sistema social que dispone de normas para su correcta utilización en contextos correctos.

Actualmente, se habla de la necesidad de que las nuevas generaciones aprendan a desarrollar habilidades, estrategias cognitivas y metacognitivas que les permitan concretar aprendizajes. Entre las básicas se encuentra la comprensión lectora, para entender que es y en que consiste, revisaremos algunas concepciones.

De la investigación de Pearson (citado por Pinzas, 1997, p. 66) "el aprendizaje de la lectura y su comprensión es un complejo proceso interactivo en el cual el lector va cambiando su centro de atención desde un procesamiento basado en el texto a uno basado en el lector". La oscilación entre el lector y el texto y viceversa dependerían principalmente del lector de sus propósitos, familiaridad con el tema, interés, motivación y tipo de discurso.

Para Alliende y Condemarín (1982, p. 14), la comprensión del lenguaje escrito es una de las formas más complejas que asume el comprender. De hecho, toda lectura no llega a un ser en blanco, sino a un ser que tiene una base comprensiva en la que se debe insertar. Siendo distinta la base comprensiva de cada ser humano, se tendría que llegar a la conclusión de que la comprensión de una lectura nunca será igual. Sera siempre diversa y subjetiva. Es muy probable que sea así. Pero, junto con esta base comprensiva personal, existe en los seres humanos la posibilidad de utilizar un conjunto de categorías intersubjetivas que son el único medio de comunicación entre seres cuyas conciencias y cuyas comprensiones globales son distintas.

Pisa (2009, p. 37), define la comprensión lectora como: "Comprender, utilizartextos escritos, reflexionar sobre ellos e implicarse sobre ellos para alcanzar los propios objetivos, desarrollar el propio conocimiento y potencial, y participar en la sociedad". Se desprende que comprender es construir el significado del texto, puede tratarse de algo básico como entender el significado de las palabras, o puede ser tan complejo como aprehender un extenso argumento o narración.

Se concluye que la comprensión lectora es compleja y abarca una amplia gama de capacidades cognitivas, desde la decodificación básica al 


\section{UNIFÉ - EPG}

conocimiento de las palabras, la gramática y la estructura y características lingüísticas y textuales, hasta el conocimiento sobre el mundo. También incluye competencias metacognitivas: la conciencia y la capacidad de utilizar diversas estrategias apropiadas para procesar textos.

Hablar de comprensión lectora es referirse a los distintos modelos que abordan el problema de esta como un proceso multinivel; esto es, el texto debe ser analizado en varios niveles que van desde los grafemas hasta el texto como un todo. Sin embargo, no hay un consenso general por lo que se refiere al modo en que estos niveles se relacionan funcionalmente.

Con base en estas características se pueden distinguir tres tipos de modelos que han ido sucediendo en el tiempo: modelos de procesamiento ascendente, modelos de procesamiento descendente y modelos interactivos (Adams, 1982 citado por Alonso, y Mateos, 1985, pp. 5-7).

El modelo ascendente también llamado de abajo hacia arriba (bottom-up), concibe la lectura como un proceso secuencial y jerárquico, que se inicia con los estímulos visuales (gráficos, lexicales, sintácticos, semánticos, contextuales y estructurales) del texto leído y terminan con la reconstrucción, interpretación que el lector hace sobre el texto.

Este modelo postula que leer equivale a realizar, en primer lugar la conversión de los signos gráficos en palabras con sentido, asociar después éstas entre sí formando frases y, finalmente, asociar las frases así construidas.

Por otra parte, el modelo descendente también llamado de arriba hacia abajo (top-down), sostiene que el lector parte de una configuración global (palabras, frases, fragmentos) y en sentido descendente va analizando sus constituyentes, sin que ello signifique que el lector logre comprender los textos que se le proponen.

El proceso se inicia en el lector (procesos cognitivos, experiencias previas, etc.) y termina en el texto, es decir que los niveles superiores interactúan con los inferiores y dirigen el flujo de información a través de ellos, por consecuencia: mayoritariamente el sentido está dado por los aportes que el lector hace al texto y no por el contenido del mismo.

$\mathrm{Si}$ la lectura fuese como asegura el modelo descendente sería improbable que se aprendiéra algo nuevo a partir de los textos; no se aumentaria el bagaje cultural si solamente se confiara en los conocimientos previos.

$Y$ finalmente, el modelo interactivo parte del supuesto que la comprensión implica procesar simultáneamente los datos explícitos en el texto y activar el conocimiento preexistente en el lector de una manera interactiva.

Este modelo propone que la información utilizada para leer proviene simultáneamente de diferentes fuentes de conocimiento (ortográfico, lexical, sintáctico y semántico) asume que existe un procesamiento paralelo entre diferentes niveles y además una comunicación bidireccional entre ellos, es decir de abajo hacia arriba y de arriba hacia abajo.

La comprensión de los textos escritos es un fenómeno muy complejo. Los factores que la determinan son muy numerosos, están mezclados entre sí y cambian constantemente. Por este motivo, las estrategias para lograr mayor comprensión lectora y las técnicas para medirla deben ser cuidadosamente analizadas.

Entrelos factores queinfluyen en la comprensión lectora están aquellos derivados del emisor (escritor), del texto en sí y los provenientes del lector.

Dentro de los factores derivados del emisor se encuentran: Conocimientos de los códigos manejados por el autor (grado de dominio del código lingüístico), conocimientos de los esquemas cognoscitivos (esquemas cognoscitivos), conocimientos del patrimonio cultural del autor y conocimientos de las circunstancias de la escritura (el lugar y el tiempo en que el libro fue escrito).

Por su parte, dentro de los factores provenientes del texto están: Los Factores físicos que influyen en su legibilidad (tamaño y nitidez de la letra), factores lingüísticos (cierre semántico, unidad, coherencia, estructuración semántica y formal, carácter pragmático y uso adecuado de elementos de cohesión). 
Alétheia 2014, 2 (1) 91

Finalmente, los factores provenientes del lector aciertan específicamente con los del emisor puesto como ya se indicó anteriormente, la comprensión lectora es una interacción entre ambos.

\section{Método}

La investigación es descriptiva (Van Dalen y Meyer, 1996) y correlacional (Sánchez y Reyes, 2006) porque pretende describir la relación existente entre las dos variables de estudio que corresponden a los componentes lingüísticos y la comprensión de lectura.

Población y muestra: La población estuvo constituida por todos los estudiantes delas instituciones educativas particulares, estatales y parroquiales, más representativas ubicadas en el distrito de Bellavista, perteneciente a la Dirección Regional de Educación del Callao que cursaban el sexto grado de educación primaria en el año 2009, con una muestra no probabilística e intencional de 271 estudiantes.

Instrumentos: El primer instrumento aplicado en el estudio es la Prueba de Complejidad Lingüística Progresiva (CLP) Formas Paralelas. Nivel 6 - Forma A. Elaborada y estandarizada en Chile (1991) por Condemarín, Alliende y Milicic; y adaptada en Perú (2005) por Delgado y cols. La prueba está dividida en ocho niveles de lectura. Cada nivel está constituido de modo que presenta una dificultad creciente desde el punto de vista lingüístico, produciéndose paralelamente un incremento en la dificultad de la comprensión, es de aplicación colectiva.

El segundo instrumento aplicado en el estudio es la Batería de Lenguaje Objetiva y Criterial BLOC-S, prueba adaptada a la realidad peruana con el objetivo de detectar las conductas comunicativas y lingüísticas alteradas de la población infantil.

Esta prueba realiza una exploración rápida de los cuatro componentes del lenguaje: morfología, sintaxis, semántica y pragmática, es de aplicación individual.

Procesamiento: Luego de la aplicación y calificación, se elaboró la base de datos para ser procesados estadísticamente en Excel.
Para identificar los estadísticos a utilizar, se aplicó la prueba de normalidad de Kolmogorov-Smirnof. Se observa que los datos están dentro de la curva de normalidad, excepto los datos del componente pragmático. Por esta razón se procesaron los datos con el estadístico de Pearson, y para complementar en el caso del componente pragmático se usó el estadístico de Spearman.

Se determinaron los niveles en base a los puntajes, que se presentan en la siguiente tabla:

Tabla 1

Rangos percentilares y puntuaciones de eneagrama para determinar los niveles nde desempeño en las pruebas aplicadas.

\begin{tabular}{ccc}
\hline & BLOC y CLP & \\
\hline PC & Eneagrama & Nivel \\
\hline 97 a 100 & 8 & Muy alto \\
79 a 96.5 & $7 \mathrm{y} 8$ & Alto \\
26 a 78 & $4-5$ y 6 & Meido \\
5 a 25 & 2 y 3 & Bajo \\
1 a 4.5 & 1 & Deficiente \\
\hline
\end{tabular}

\section{Resultados}

\section{Según Institución Educativa}

Al asociar de manera general el nivel de la comprensión lectora y los componentes lingüísticos, según el total de estudiantes, de las instituciones educativas evaluadas se observan los siguientes resultados. 


\section{UNIFÉ - EPG}

Tabla 2

Valores de la Media Aritmética, Coeficiente de Correlación, Zeta Empirico y Teórico, Criterio y Decisión de la relación entre el nivel de comprensión lectora y el nivel de global de los componentes lingüisticos, según las instituciones educativas estatal, parroquial yparticular.

\begin{tabular}{|c|c|c|c|c|c|c|c|}
\hline $\begin{array}{l}\text { Institucion } \\
\text { educativa }\end{array}$ & & $\mathrm{X}$ & $\mathrm{r}$ & $\mathrm{Z}_{\mathrm{c}}$ & Z & Criterio & Decisión \\
\hline \multirow{2}{*}{ Estatal } & $\begin{array}{c}\text { Comprensión } \\
\text { Lectora }\end{array}$ & 17.16 & \multirow{2}{*}{0.44} & \multirow{2}{*}{3.99} & \multirow{4}{*}{1.96} & \multirow{4}{*}{$\begin{array}{l}\text { "Si } \mathrm{Z}_{\mathrm{c}} \geq \mathrm{Z}=> \\
\text { Rechazar } \mathrm{H}_{\mathrm{o}} "\end{array}$} & \multirow{2}{*}{ Rechazar H } \\
\hline & $\begin{array}{l}\text { Componentes } \\
\text { Lingüisticos }\end{array}$ & 69.04 & & & & & \\
\hline \multirow[t]{2}{*}{ Parroquial } & $\begin{array}{l}\text { Comprensión } \\
\text { Lectora }\end{array}$ & 20.37 & \multirow{2}{*}{0.49} & \multirow{2}{*}{4.70} & & & \multirow[b]{2}{*}{ Rechazar $\mathrm{H}_{\mathrm{c}}$} \\
\hline & $\begin{array}{l}\text { Componentes } \\
\text { Lingüisticos }\end{array}$ & 80.83 & & & & & \\
\hline \multirow{2}{*}{ Particular } & $\begin{array}{l}\text { Comprensión } \\
\text { Lectora }\end{array}$ & 24.82 & \multirow{2}{*}{0.47} & \multirow{2}{*}{4.62} & & & \multirow{2}{*}{ Rechazar $\mathrm{H}_{\mathrm{o}}$} \\
\hline & $\begin{array}{c}\text { Componentes } \\
\text { Lingüisticos } \\
\end{array}$ & 66.04 & & & & & \\
\hline
\end{tabular}

Al relacionar, mediante el test de Pearson, los coeficientes de correlación son 0.44, 0.49 y 0.47 (nivel medio) respectivamente en cada institución.

Cada uno de los z empíricos de las instituciones educativas: 3.99 (Estatal) 4.70 (Parroquial) y 4.62 (Particular), cumplen con ser mayor o igual que el z teórico (1.96) por lo que se rechaza la hipótesis nula; es decir, que sí existe correlación significativa entre las variables en estudio; siendo que, a mayor puntaje en los componentes lingüísticos los estudiantes obtienen también mayor puntaje en comprensión lectora. Se verifica el cumplimiento de la hipótesis general H.1

$\mathrm{Al}$ asociar el nivel de la comprensión lectora por cada uno de los componentes lingüísticos (morfológico, sintáctico, semántico y pragmático), según las instituciones educativas evaluadas se observan los siguientes resultados.

Tabla 3

Valores de la Media Aritmética, Coeficiente de Correlación, Zeta Empirico y Teórico, Criterio y Decisión de la correlación entre el nivel de comprensión lectora y el nivel del componente morfológico, de las instituciones educativas estatal, parroquialy particular

\begin{tabular}{|c|c|c|c|c|c|c|c|}
\hline $\begin{array}{c}\text { Institucion } \\
\text { educativa }\end{array}$ & & M & $\mathrm{r}$ & $\mathrm{Z}_{\mathrm{c}}$ & Z & Criterio & Decisión \\
\hline \multirow{2}{*}{ Estatal } & $\begin{array}{l}\text { Comprensión } \\
\text { Lectora }\end{array}$ & 17.16 & \multirow{2}{*}{0.37} & \multirow{2}{*}{3.34} & \multirow[b]{4}{*}{1.96} & \multirow{4}{*}{$\begin{array}{l}\text { "Si } Z_{c} \geq Z_{. .}=> \\
\text {Rechazar } H_{o} "\end{array}$} & \multirow{2}{*}{ Rechazar $\mathrm{H}_{\mathrm{o}}$} \\
\hline & $\begin{array}{c}\text { Componentes } \\
\text { Morfológicos }\end{array}$ & 20.62 & & & & & \\
\hline \multirow{2}{*}{ Parroquial } & $\begin{array}{l}\text { Comprensión } \\
\text { Lectora }\end{array}$ & 20.37 & \multirow[b]{2}{*}{0.35} & \multirow{2}{*}{3.32} & & & \multirow[b]{2}{*}{ Rechazar $\mathrm{H}_{\mathrm{o}}$} \\
\hline & $\begin{array}{l}\text { Componentes } \\
\text { Morfológicos }\end{array}$ & 24.20 & & & & & \\
\hline \multirow{2}{*}{ Particular } & $\begin{array}{l}\text { Comprensión } \\
\text { Lectora }\end{array}$ & 24.82 & \multirow{2}{*}{0.32} & \multirow{2}{*}{3.11} & & & \multirow{2}{*}{ Rechazar $\mathrm{H}_{\mathrm{o}}$} \\
\hline & $\begin{array}{c}\text { Componentes } \\
\text { Morfológicos }\end{array}$ & 23.33 & & & & & \\
\hline
\end{tabular}


Al relacionar, mediante el test de Pearson, los coeficientes de correlación son 0.37, 0.35 y 0.32 (niveles bajos) respectivamente.

Cada uno de los z empíricos de las instituciones educativas: 3.34 (Estatal) 3.32 (Parroquial) y 3.11 (Particular), cumplen con ser mayor o igual que el z teórico (1.96) por lo que se rechaza la hipótesis nula; es decir, que sí existe correlación significativa entre las variables en estudio; siendo que, a mayor puntaje en el componente morfológico los estudiantes obtienen también mayor puntaje en comprensión lectora. Se verifica el cumplimiento de la hipótesis específica H.1.1

Tabla 4

Valores de la Media Aritmética, Coeficiente de Correlación, Zeta Empirico y Teórico, Criterio y Decisión de la correlación entre el nivel de comprensión lectora el nivel del componente sintáctico, de las instituciones educativas estatal, parroquial y particular

\begin{tabular}{|c|c|c|c|c|c|c|c|}
\hline $\begin{array}{l}\text { Institucion } \\
\text { educativa }\end{array}$ & & M & $\mathrm{r}$ & $\mathrm{Z}_{\mathrm{c}}$ & Z & Criterio & Decisión \\
\hline \multirow{2}{*}{ Estatal } & $\begin{array}{l}\text { Comprensión } \\
\text { Lectora }\end{array}$ & 17.16 & \multirow{2}{*}{0.41} & \multirow{2}{*}{3.69} & \multirow{4}{*}{1.96} & \multirow{4}{*}{$\begin{array}{l}\text { "Si } \mathrm{Z}_{\mathrm{c}} \geq \mathrm{Z} . .= \\
\text { Rechazar } \mathrm{H}_{\mathrm{o}} \text { " }\end{array}$} & \multirow{2}{*}{ Rechazar $\mathrm{H}_{\mathrm{o}}$} \\
\hline & $\begin{array}{l}\text { Componentes } \\
\text { Morfológicos }\end{array}$ & 15.98 & & & & & \\
\hline \multirow[t]{2}{*}{ Parroquial } & $\begin{array}{c}\text { Comprensión } \\
\text { Lectora }\end{array}$ & 20.37 & \multirow{2}{*}{0.36} & \multirow{2}{*}{3.48} & & & \multirow[b]{2}{*}{ Rechazar $\mathrm{H}_{\mathrm{o}}$} \\
\hline & $\begin{array}{l}\text { Componentes } \\
\text { Morfológicos }\end{array}$ & 22.57 & & & & & \\
\hline \multirow{3}{*}{ Particular } & $\begin{array}{c}\text { Comprensión } \\
\text { Lectora }\end{array}$ & 24.82 & \multirow{3}{*}{0.30} & \multirow{3}{*}{2.97} & & & \multirow{3}{*}{ Rechazar $\mathrm{H}_{\mathrm{o}}$} \\
\hline & Componentes & & & & & & \\
\hline & Morfológicos & 12.79 & & & & & \\
\hline
\end{tabular}

Al relacionar, mediante el test de Pearson, los coeficientes de correlación son 0.41 (nivel medio), 0.36 (nivel bajo) y 0.30 (nivel bajo) respectivamente.

Cada uno de los z empírico de las instituciones educativas es de 3.69 (Estatal), 3.48 (Parroquial) y 2.97 (Particular), cumplen con ser mayor o igual que el z teórico (1.96) por lo que se rechaza la hipótesis nula; es decir, que sí existe correlación significativa entre las variables en estudio; siendo que, a mayor puntaje en el componente sintáctico los alumnos obtienen también mayor puntaje en comprensión lectora. Se verifica el cumplimiento de la hipótesis específica H.1.2 


\section{UNIFÉ - EPG}

Tabla 5

Valores de la Media Aritmética, Coeficiente de Correlación, Zeta Empirico y Teórico, Criterio y Decisión de la correlación entre el nivel de comprensión lectora y el nivel del componente semántico, de las instituciones educativas estatal, parroquialy particular

\begin{tabular}{|c|c|c|c|c|c|c|c|}
\hline $\begin{array}{l}\text { Institucion } \\
\text { educativa }\end{array}$ & & M & $\mathrm{r}$ & $\mathrm{Z}_{\mathrm{c}}$ & Z & Criterio & Decisión \\
\hline \multirow{2}{*}{ Estatal } & Comprensión Lectora & 17.16 & \multirow{2}{*}{0.27} & \multirow{2}{*}{2.39} & \multirow{6}{*}{1.96} & \multirow{6}{*}{$\begin{array}{r}\text { "Si } \mathrm{Z}_{\mathrm{c}} \geq \mathrm{Z} . . \\
=> \\
\text { Rechazar } \mathrm{H}_{\mathrm{o}}\end{array}$} & \multirow{2}{*}{ Rechazar $\mathrm{H}_{\mathrm{o}}$} \\
\hline & ComponentePragmatico & 17.15 & & & & & \\
\hline \multirow[t]{3}{*}{ Parroquial } & Comprensión Lectora & 20.37 & \multirow{2}{*}{0.39} & \multirow{3}{*}{3.68} & & & \multirow{3}{*}{ Rechazar $\mathrm{H}_{\mathrm{o}}$} \\
\hline & ComponentePragmatico & 17.43 & & & & & \\
\hline & Comprensión Lectora & 24.82 & \multirow[b]{2}{*}{0.27} & & & & \\
\hline Particular & ComponentePragmatico & 15.28 & & 2.60 & & & Rechazar $\mathrm{H}_{\mathrm{o}}$ \\
\hline
\end{tabular}

Al relacionar, mediante el test de Pearson, los coeficientes de correlación son 0.27, 0.39 y 0.27 (niveles bajos) respectivamente.

Cada uno de los z empíricos de las instituciones educativas: 2.39 (Estatal) 3.68 (Parroquial) y 2.60 (Particular), cumplen con ser mayor o igual que el z teórico (1.96) por lo que se rechaza la hipótesis nula; es decir, que sí existe relación significativa entre las variables en estudio; siendo que, a mayor puntaje en el componente semántico los alumnos obtienen también mayor puntaje en comprensión lectora. Se verifica el cumplimiento de la hipótesis específica H.1.3.

Tabla 6

Valores de la Media Aritmética, Coeficiente de Correlación, Zeta Empirico y Teórico, Criterio y Decisión de la relación entre el nivel de comprensión lectora y el nivel del componente pragmático, de las instituciones educativas estatal, parroquialy particular

\begin{tabular}{|c|c|c|c|c|c|c|c|c|}
\hline $\begin{array}{l}\text { Institucion } \\
\text { educativa }\end{array}$ & & $\mathrm{X}$ & $\mathrm{r}_{\mathrm{p}}$ & $r_{s}$ & $\mathrm{Z}_{\mathrm{c}}$ & Z & Criterio & Decisión \\
\hline & Comprensión Lectora & 17.16 & & & & \multirow{6}{*}{1.96} & \multirow{6}{*}{$\begin{array}{l}\text { "Si Z }{ }_{c} \geq Z_{. .}=> \\
\text {Rechazar } H_{0} "\end{array}$} & \multirow[b]{2}{*}{ Rechazar $\mathrm{H}_{\mathrm{o}}$} \\
\hline Estatal & $\begin{array}{l}\text { Componente } \\
\text { Pragmatico }\end{array}$ & 15.29 & 0.37 & .300 & 3.31 & & & \\
\hline \multirow{2}{*}{ Parroquial } & Comprensión Lectora & 20.37 & & & & & & \\
\hline & $\begin{array}{l}\text { Componente } \\
\text { Pragmatico }\end{array}$ & 16.57 & 0.56 & .513 & 5.33 & & & Rechazar $\mathrm{H}_{\mathrm{o}}$ \\
\hline \multirow[b]{2}{*}{ Particular } & Comprensión Lectora & 24.82 & \multirow[b]{2}{*}{0.38} & \multirow[b]{2}{*}{.426} & \multirow[b]{2}{*}{3.72} & & & \multirow[b]{2}{*}{ Rechazar $\mathrm{H}_{\mathrm{o}}$} \\
\hline & $\begin{array}{c}\text { Componente } \\
\text { Pragmatico }\end{array}$ & 15.03 & & & & & & \\
\hline
\end{tabular}

Al relacionar, mediante el test de Pearson y Spearman, los coeficientes de correlación de 0.37 y .300 (nivel bajo), 0.56 y .513 (nivel medio) y 0.38 y .426 (nivel bajo) respectivamente.

Cada uno de los z empíricos de las instituciones educativas: 3.31 (Estatal) 5.33 (Parroquial) y 3.72 (Particular), cumplen con ser mayor o igual que el z teórico (1.96) por lo que se rechaza la hipótesis nula; es decir, que sí existe correlación significativa entre las variables en estudio; siendo que, a mayor puntaje en el componente pragmático los estudiantes obtienen también mayor puntaje en comprensión lectora. Se verifica el cumplimiento de la hipótesis específica H.1.4 


\section{Según género}

$\mathrm{Al}$ asociar de manera general el nivel de la comprensión lectora y los componentes lingüísticos, según género, de las instituciones educativas evaluadas se observan los siguientes resultados.

Tabla 7

Valores de la Media Aritmética, Coeficiente de Correlación, Zeta Empirico y Teórico, Criterio y Decisión de la relación entre el nivel de comprensión lectora y el nivel global de los componentes lingüísticos, de los estudiantes de sexto grado de primaria, según género.

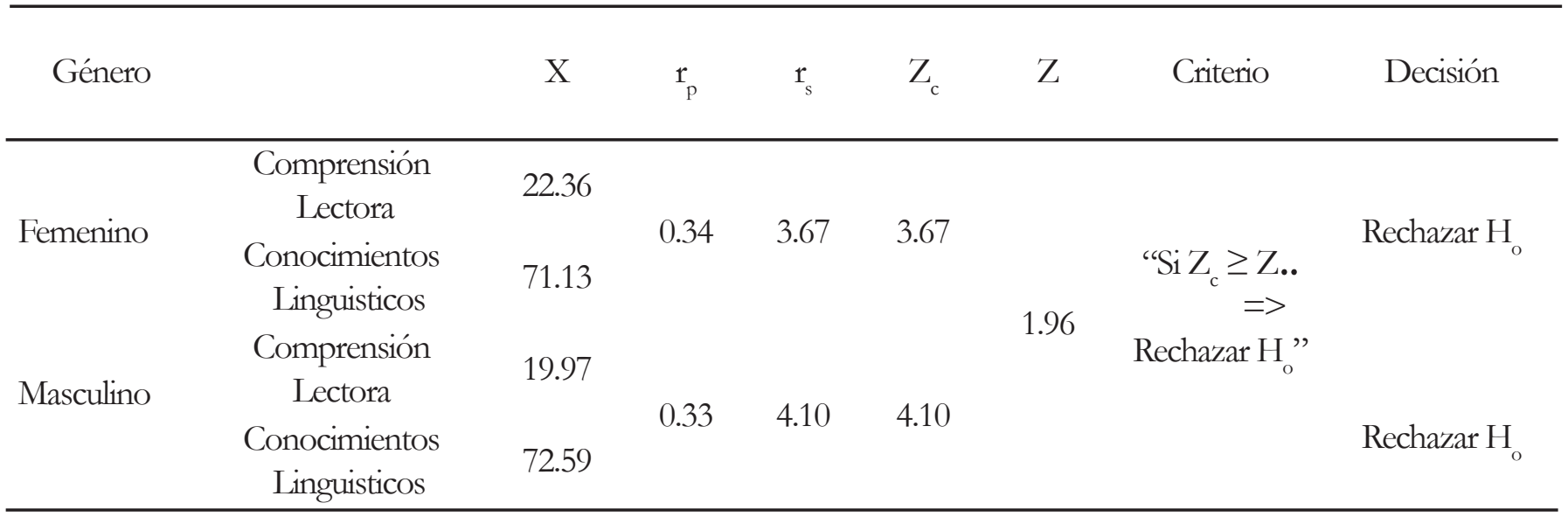

Al relacionar, mediante el test de Pearson, los coeficiente de correlación son 0.34 y 0.33 (niveles bajos) respectivamente.

El z empírico de las mujeres es de 3.67 y el de los varones de 4.10, ambos cumplen con ser mayor o igual que el z teórico (1.96) por lo que se rechaza la hipótesis nula; es decir, que sí existe correlación significativa entre las variables en estudio; siendo que, a mayor puntaje en los componentes lingüísticos los estudiantes tanto de género femenino y masculino obtienen también mayor puntaje en comprensión lectora. Se verifica el cumplimiento de la hipótesis general H.2

$\mathrm{Al}$ asociar el nivel de la comprensión lectora por cada uno de los componentes lingüísticos (morfológico, sintáctico, semántico y pragmático), según género se observan los siguientes resultados.

Tabla 8

Valores de la Media Aritmética, Coeficiente de Correlación, Zeta Empirico y Teórico, Criterio y Decisión de correlación entre el nivel de comprensión lectora y el nivel del componente morfológico, según género.

\begin{tabular}{|c|c|c|c|c|c|c|c|}
\hline Género & & $\mathrm{X}$ & $r_{p}$ & $Z_{c}$ & Z & Criterio & Decisión \\
\hline \multirow{2}{*}{ Femenino } & Comprensión Lectora & 22.36 & 047 & 500 & \multirow{4}{*}{1.96} & \multirow{4}{*}{$\begin{array}{c}\text { "Si } Z_{\mathrm{c}} \geq \mathrm{Z..} \\
=> \\
\text { Rechazar } \mathrm{H}_{\mathrm{o}},\end{array}$} & \multirow{3}{*}{ Rechazar $\mathrm{H}_{\mathrm{o}}$} \\
\hline & Componente Morfológico & 23.09 & $0.4 /$ & 5.08 & & & \\
\hline \multirow{2}{*}{ Masculino } & Comprensión Lectora & 19.97 & \multirow{2}{*}{0.38} & \multirow{2}{*}{4.02} & & & \\
\hline & Componente Morfológico & 22.59 & & & & & Rechazar $\mathrm{H}_{\mathrm{o}}$ \\
\hline
\end{tabular}

$\mathrm{Al}$ relacionar, mediante el test de Pearson, los coeficiente de correlación de 0.47 (nivel medio) y 0.38 (nivel bajo), respectivamente

El z empírico de las estudiantes de género femenino es 5.08 y el de los estudiantes del género masculino es 4.02, ambos cumplen con ser mayor o igual que el z teórico (1.90) por lo que se rechaza la hipótesis nula; es decir, que sí existe correlación significativa entre las variables en estudio; siendo que, a mayor puntaje en el componente 


\section{UNIFÉ - EPG}

morfológico obtienen también los estudiantes mayor puntaje en comprensión lectora. Se verifica el cumplimiento de la hipótesis específica H 2.1

Tabla 9

Valores de la Media Aritmética, Coeficiente de Correlación, Zeta Empirico y Teórico, Criterio y Decisión de correlación entre el nivel de comprensión lectora y el nivel del componente sintáctico, según género.

\begin{tabular}{|c|c|c|c|c|c|c|c|}
\hline Género & & $\mathrm{X}$ & $\mathrm{r}_{\mathrm{p}}$ & $Z_{c}$ & Z & Criterio & Decisión \\
\hline \multirow{2}{*}{ Femenino } & Comprensión Lectora & 22.36 & \multirow{2}{*}{0.18} & \multirow{2}{*}{1.95} & & \multirow{2}{*}{$\begin{array}{c}\text { "Si Z }{ }_{c} \geq Z . \\
=>\end{array}$} & Falla en \\
\hline & Componente sintáctico & 15.97 & & & & & Rechazar $\mathrm{H}_{\mathrm{o}}$ \\
\hline \multirow[t]{2}{*}{ Masculino } & Comprensión Lectora & 19.97 & \multirow{2}{*}{0.15} & \multirow{2}{*}{1.87} & 1.96 & Rechazar Ho" & Falla en \\
\hline & Componente sintáctico & 17.90 & & & & & Rechazar $\mathrm{H}_{\mathrm{o}}$ \\
\hline
\end{tabular}

$\mathrm{Al}$ relacionar, mediante el test de Pearson, los coeficientes de correlación de 0.18 y 0.15 (niveles bajos). $\mathrm{El} \mathrm{z}$ empírico de las estudiantes de género femenino es 1.95 y el de los estudiantes de género masculino es 1.87, cumplen con no ser mayor o igual que el z teórico (1.96) por lo que se falla en rechazar la hipótesis nula; es decir, que no existe correlación significativa entre las variables en estudio; según género. No se verifica el cumplimiento de la hipótesis específica H 2.2

Tabla 10

Valores de la Media Aritmética, Coeficiente de Correlación, Zeta Empirico y Teórico, Criterio y Decisión de correlación entre el nivel de comprensión lectora y el nivel del componente semántico, según género.

\begin{tabular}{|c|c|c|c|c|c|c|c|}
\hline Género & & $\mathrm{X}$ & $r_{p}$ & $\mathrm{Z}_{\mathrm{c}}$ & Z & Criterio & Decisión \\
\hline \multirow{2}{*}{ Femenino } & Comprensión Lectora & 22.36 & 017 & 187 & \multirow{4}{*}{1.96} & \multirow{4}{*}{$\begin{array}{l}\text { "Si Z }{ }_{c} \geq Z_{. .}=> \\
\text {Rechazar } \mathrm{H}_{\mathrm{o}} "\end{array}$} & Falla en \\
\hline & Componente Semántico & 16.58 & 0.17 & 1.81 & & & Rechazar $\mathrm{H}_{\mathrm{o}}$ \\
\hline \multirow[t]{2}{*}{ Masculino } & Comprensión Lectora & 19.97 & \multirow{2}{*}{0.14} & \multirow{2}{*}{1.78} & & & Falla en \\
\hline & Componente Semántico & 16.57 & & & & & Rechazar H \\
\hline
\end{tabular}

Al relacionar, mediante el test de Pearson, los coeficiente de correlación son 0.17 y 0.14 (niveles bajos). $\mathrm{El} \mathrm{z}$ empírico de las estudiantes del género femenino es 1.87 y el de los estudiantes del género masculino es 1.78, cumplen con no ser mayor o igual que el z teórico (1.96) por lo que se falla en rechazar la hipótesis nula; es decir, que no existe correlación significativa entre las variables en estudio; según género. No se verifica el cumplimiento de la hipótesis específica H 2.3 
Alétheia 2014,2 (1) 97

Tabla 11

Valores de la Media Aritmética, Coeficiente de Correlación, Zeta Empirico y Teórico, Criterio y Decisión de correlación entre el nivel de comprensión lectora y el nivel del componente pragmático, según género.

\begin{tabular}{|c|c|c|c|c|c|c|c|c|}
\hline Género & & M & $\mathrm{r}_{\mathrm{p}}$ & $\mathrm{r}_{\mathrm{s}}$ & $\mathrm{Z}_{\mathrm{c}}$ & Z & Criterio & Decisión \\
\hline Femenino & $\begin{array}{c}\text { Comprensión } \\
\text { Lectora } \\
\text { Componente } \\
\text { Pragmático }\end{array}$ & 22.36 & 0.26 & .216 & 2.74 & & $\begin{array}{c}\text { "Si } Z_{\mathrm{c}} \geq \mathrm{Z..} \\
=>\end{array}$ & Rechazar $\mathrm{H}_{\mathrm{o}}$ \\
\hline Masculino & $\begin{array}{c}\text { Comprensión } \\
\text { Lectora } \\
\text { Componente } \\
\text { Pragmático }\end{array}$ & 19.97 & 0.41 & .367 & 5.74 & 1.96 & Rechazar Ho" & Rechazar $\mathrm{H}_{\mathrm{o}}$ \\
\hline
\end{tabular}

Al relacionar, mediante el test de Pearson y Spearman, los coeficientes de correlación de 0.26 y .216 (nivel bajo) y 0.41 y .367 (nivel medio), respectivamente.

El z empírico de los estudiantes del género femenino es de 2.74 y el de los estudiantes del género masculino 5.14, ambos cumplen con ser mayor o igual que el z teórico (1.96) por lo que se rechaza la hipótesis nula; es decir, que sí existe correlación significativa entre las variables en estudio. Se verifica el cumplimiento de la hipótesis específica H 2.4

\section{Discusión}

Existe correlación entre los componentes lingüísticos y la comprensión lectora del total de estudiantes; siendo que a mayor puntaje en los componentes lingüísticos los estudiantes obtienen también mayor puntaje en comprensión lectora.

Así también, teniendo en cuenta las tres instituciones educativas se encontró que sí existe correlación entre las variables de estudio; siendo que, a mayor puntaje en los componentes lingǘsticos los estudiantes obtienen también mayor puntaje en comprensión lectora.

Al respecto, Acosta (2010, p. 180) menciona que:

Parece claro que la adquisición de la lectura se apoya en el lenguaje. Por tanto, dada la estrecha relación existente entre las habilidades lingüísticas y la lectura, no resulta sorprendente que los niños con dificultades del lenguaje tengan también dificultades en el aprendizaje de la lectura

En este sentido, se afirma que el desarrollo adecuado y oportuno de las habilidades lingüísticas en general conlleva a un buen desarrollo de la lectura y por ende la comprensión lectora.

Así también, luego de asociar los componentes lingüísticos y la comprensión lectora, según el tipo de institución educativa, se encontró que existe relación significativa entre las variables en estudio, siendo la institución educativa parroquial la que tuvo mejor rendimiento que la institución particular seguida de la institución estatal.

En nuestro país, existe una asociación significativa entre el tipo de escuela al que asisten los niños y el nivel socioeconómico de las familias, en el sentido de que las escuelas de gestión pública suelen contar con una matrícula de niveles socioeconómicos bajos, en tanto que a las escuelas privadas concurren estudiantes de niveles socioeconómicos medio - altos (Ansion, 2011). Los niños de este nivel socioeconómico suelen rendir mejor en este tipo de pruebas que los niños de niveles socioeconómicos bajos (Plaza, 2001). En este 


\section{UNIFÉ - EPG}

sentido, los resultados se relacionan con lo anterior, ya que los factores sociales y económicos poseen un impacto considerable en la adquisición de habilidades lingüísticas y de lectura (Lonigan \& cols., 1998; Raz \& Bryant, 1990).

$\mathrm{Al}$ asociar los componentes lingüísticos y la comprensión lectora, del total de los estudiantes de sexto grado de educación primaria según género, se encontró que sí existe correlación entre el nivel global de los componentes lingüísticos y el nivel de comprensión lectora.

Probablemente, como Belinchón, Riviere e Igoa (1994) señalan, que al emplear el lenguaje en interacciones tan sencillas como la indicada, los interlocutores ponen en juego un amplio conjunto de conocimientos compartidos.

Debemos tener en cuenta, no obstante, que el lenguaje no se percibe en forma de unidades fragmentadas, sino como una sucesión continua de elementos perceptivos, es decir, si bien parece cierto que las etapas iniciales de la percepción del lenguaje consta de procesos casi exclusivamente dirigidos por las propiedades de la señal (de "abajo-arriba"), también hay que tomar en consideración otros factores contextuales (representaciones léxicas, estructuras sintácticas y conocimiento semántico) que guían, a partir de un determinado momento, estas operaciones perceptivas (p. 320).

En suma, los interlocutores necesitan emplear un amplio conjunto de conocimientos generales, de inferencias y principios sobre el mundo interno e intencional de las personas. Así que para mantener un intercambio comunicativo no es preciso sólo poseer habilidad lingüística, sino que también es necesario dominar otras habilidades, tales como saber respetar la alternancia de turnos, adaptarse al interlocutor, utilizar el contexto intralingüístico, el conocimiento general sobre el mundo, etc., que están relacionadas con el desarrollo cognitivo y social.

\section{Conclusiones}

1. Existe correlación entre el nivel global de los componentes lingüísticos y el nivel de comprensión lectora en los estudiantes del sexto grado de educación primaria según tipo de institución educativa.

2. El componente morfológico y el nivel de comprensión lectora en los estudiantes del sexto grado de educación primaria se correlacionan según tipo de institución educativa.

3. Entre el componente sintáctico y el nivel de comprensión lectora existe correlación en los estudiantes del sexto grado de educación primaria, según tipo de institución.

4. Entre el componente semántico y el nivel de comprensión lectora existe correlación en los estudiantes del sexto grado de educación primaria, según tipo institución educativa.

5. Entre el componente pragmático y el nivel de comprensión lectora se encontró correlación en los estudiantes del sexto grado de educación primaria, según tipo de institución educativa.

6. Existe correlación entre el nivel global de los componentes lingüísticos y el nivel de comprensión lectora en los estudiantes del sexto grado de educación primaria según género.

7. El componente morfológico y el nivel de comprensión lectora en los estudiantes del sexto grado de educación primaria se correlacionan según género.

8. Entre el componente sintáctico y el nivel de comprensión lectora no existe correlación por género.

9. Entre el componente semántico y el nivel de comprensión lectora no existe correlación según género.

10. Entre el componente pragmático y el nivel de comprensión lectora se encontró correlación en los estudiantes del sexto grado de educación primaria, según género. 


\section{Referencias}

Acosta, V. y Moreno, A. (1999). Dificultades del lenguaje en ambientes educativos. Barcelona, España: Masson, S.A.

Acosta,V. y Moreno, A. (2010). Dificultades del lenguaje e inclusión educativa: manual para logopedas, psicólogos y profesores. Barcelona, España: Lexus.

Alonso,J.y Mateos, M. (1985) Comprensión lectora. Modelos, entrenamiento y evaluación.

[On - Line] Disponible en http:/ dialnet.unirioja. es/descarga/articulo/667401.pdf

Alliende, F., y Condemarin, M. (1982). Manual de Comprensión lectora de complejidad lingiüstica progresiva. Santiago:de Chile, Chile: Universidad Católica de Chile.

Ansion (2011) Volver a Pensar la Educación Pública. Revista Peruana de Investigación Educativa. No o.3,p. 52-73. Pontificia Universidad Católica del Perú (PUCP) Belinchón, Riviere e Igoa (1994). Psicología del Lenguaje: Investigación y teoría. (2a. edic.) Madrid, España: Trotta

Berk, L. (1999). Desarrollo del niño y el adolescente (4a ed.). Madrid, España: Prentice Hall

Berko, L. y Bernstein, N. (1999). Psicolingüística. Madrid: Mc. Graw- Hill Interamericana de España, S.A.

Blondet, D. y Pinheiro, E. (2012) Efectos del Programa "CLO" para mejorar los componentes del lenguaje oral expresivo en niños con trastornos específicos del lenguaje (TEL) de 5to. y 6to. de primaria. (Tesis de Maestría inédita) Universidad Femenina del Sagrado Corazón Lima, Perú.

Condemarín, M.; Milicic, N. y Alliende, F. (1991). Prueba CLP-Formas paralelas. Santiago de Chile: Universidad Católica de Chile.

Delgado, A. y cols. (2005). Adaptación de la Prueba de Comprensión Lectora de Complejidad Lingüistica Progresiva. Nivel 6 - Forma A (CLP 6-A) Lima: UNMSM.

Galeote, M. (2002). Adquisición del lenguaje: problemas, imvestigación y perspectivas. Madrid: Pirámide.

Informe PISA (2009). Lo que los estudiantes saben y pueden hacer. Rendimiento de los estudiantes en lectura, matemáticas y ciencias. Madrid, España: OCDE
Alétheia 2014, 2 (1) 99

Programa para la evaluación internacional de los alumnos.

Lonigan, J., Burguess, S., Anthhony, J. and Barket, T. (1998). Development of phonological sensitivity in 2-to 5-year-old chidren. Journal of Educational Psychology, 90, 294-311.

Miretti, M. (2006). La lengua oral en la educación inicial. Santa Fé de Bogota. Colombia: Homo Sapiens.

Muñoz, A. (2010). Psicología del desarrollo en la etapa de educación primaria. Madrid, España: Pirámide

Niño, V. (2002). Semiótica y Lingüistica Aplicadas al español ( $4^{\mathrm{a}}$ ed.). Bogotá, Colombia: ECOE

Owens, R. (2003). Desarrollo del Lenguaje (5aed.). Madrid, España: Pearson Educación

Pinzás, J. (1997). Metacognición y lectura. Lima, Perú: Pontificia Universidad Católica del Perú.

Plaza, M. (2001). The interaction between phonological processing, syntactic awareness and reading: a longitudinal study from Kindergarten to Grade 1. First Language, 21, 003-024.

Sánchez, H. y Reyes, C. (2006). Metodología y Diseños en la Investigación Cientifica. Lima, Perú: Visión Universitaria.

Unidad de la Medición de la calidad. (2010). Sistema de consulta de resultados de la evaluación censal de estudiantes. [On - Line] Disponible en http://sistemas02. minedu.gob.pe/consulta_ece/publico/index. php

Van, D., Meyer, W. (1996). Manual de técnica de la investigación educacional. México D.F., México: Paidós. 